\title{
Use of Amount-of-Substance Terminology and Equations in Field Desorption Theory
}

\author{
Richard G. Forbes ${ }^{1}$ (1)
}

Published online: 3 August 2020

(c) The Author(s) 2020

\begin{abstract}
This note proposes that the theories of field evaporation and field desorption, as used in atom-probe microscopy and related atomic-level contexts, should be consistently formulated in terms of a set of "seven-dimensional (7-D)" formulae and equations that involve the physical quantity "amount of substance", but make use of an atomic-level constant effectively equal to "one atom" (or, more generally, "one entity"). It is argued that the term "count" should be introduced as an alternative name (more suited to atomic-level contexts) for the quantity "amount of substance". For field evaporation/desorption theories, relevant definitions and formulae are proposed, and compared with the "six-dimensional" system (based on the dimensionless quantity "number of atoms/entities") sometimes used in the literature. Advantages of using a 7-D system are noted. It is argued that there is also an increasing need for a comprehensive system of official nomenclature for atomic-level constants and units, for all three of the extensive quantities "mass", "electric charge" and "amount of substance". It is also argued that, in the longer term, considerations of the kind being proposed here for field evaporation/desorption theories might usefully be applied more generally in atomic-level rate theory.
\end{abstract}

Keywords Field evaporation theory $\cdot$ Amount of substance $\cdot$ Counting units $\cdot$ Atomic-level constants

\section{Introduction}

In the 1970s, the International Standards Organization and related organizations recommended a system of quantities and equations for use in science and engineering. Since 2009, this has been called the International System of Quantities (ISQ). The existence of this formal name is helpful in making a distinction between (a) the modern system of quantities and equations and (b) the formal system of units (the SI system) based on it. Obviously, in the ISQ there are seven base-quantities and (correspondingly) seven ISQ "dimensions" [1]. In the discussion below, the ISQ dimensions of amount of substance, of length, and of time, are denoted by $\mathbf{N}, \mathbf{L}$ and $\mathbf{T}$, respectively, in accordance with the usual convention [1].

In the ISQ, there are three core physical quantities that quantify "how much of a basic physical property is present",

Richard G. Forbes

r.forbes@trinity.cantab.net

1 Advanced Technology Institute and Department of Electrical and Electronic Engineering, University of Surrey,

Guildford GU2 7XH, Surrey, UK namely mass $(m)$, electric charge $(q)$ and amount of substance $(n)$.

In the materials science technique called atom-probe tomography (APT) [2,3], atoms are removed from a pointed specimen surface by a process called field evaporation (FEV), and are then subject to analysis using a positionsensitive time-of-flight mass spectrometer. A very similar technique ("pulsed field desorption mass spectrometry") has been used by Kruse [4, 5], in the context of catalysis, to analyse molecules adsorbed on the surface of a pointed substrate.

For simplicity, the remarks below discuss the theory of field evaporation, but similar considerations apply to the theory of field desorption and could also be applied more generally to other atomic-level rate theories.

At present, the theory of field evaporation (and related processes) is often formulated in terms of what the author calls a "six-dimensional (6-D) system". A 6-D system is one in which the ISQ quantity "amount of substance" and the related ISQ dimension $\mathbf{N}$, are not used. In a 6-D system, the physical property "rate of field evaporation" is expressed as the physical quantity "number of entities evaporated per 
unit time": this physical quantity has ISQ dimensions $\mathbf{N T}^{-1}$, and is measured in $\mathrm{s}^{-1}$.

This note derives from the author's perception that it would be better practice to use what the author calls a "seven-dimensional (7-D) system": in this system, the ISQ quantity "amount of substance" and the related ISQ dimension $\mathbf{N}$, are used, and the physical property "rate of field evaporation" is expressed as the physical quantity "amount of substance evaporated per unit time". This physical quantity has ISQ dimensions $\mathbf{N T}^{-1}$, and its SI unit would be $\mathrm{mol} / \mathrm{s}$.

This practice would be in accordance with modern understanding that the physical quantity "amount of substance" is a count of how many individual molecules (or other basic entities) of a designated chemical type are involved in some chemical or physical process, and in accordance with the modern convention that a count of this type has its own ISQ dimension. Modern understanding is that the "mole" is a very large counting unit, suitable for counting the very large number of atoms or other basic entities involved in macroscopic chemical or physical processes.

However, what APT and similar atomic-level techniques need (in the author's view) is an atomic-level variant of this modern (ISQ) approach. In this atomic-level variant, the equations (and quantities used) would be identical for very large and very small counts of basic entities, and there would be a recognised convention about use of an atomic-level unit of amount of substance. This convention would be intellectually analogous to the recent use of the "unified atomic mass unit" (u), and then the alternative name the "Dalton" (Da), as atomic-level units of mass.

The author's perception is that, informally, an atomiclevel system of this type is already partly in place, but contains linguistic and other inconsistencies. The aim of this note is to set out and discuss a consistent atomic-level system, and to deal with some related linguistic issues.

\section{Definition of the Atomic-Level Variant of the ISQ Seven-Dimensional System}

\subsection{Atomic-Level Constant and Units}

A key part of an atomic-level system of this kind is an atomic-level quantity $n_{1}$. This plays the same role for amount of substance as the unified atomic mass constant $\left(m_{\mathrm{u}}\right)$ does for mass, and the elementary (positive) charge $(e)$ does for electric charge. A formal definition of $n_{1}$ is as follows.

"The constant $n_{1}$ denotes the amount of substance of a system that contains one entity. The nature of the entity must be specified, and may be an atom, a molecule, an ion, an electron, another elementary entity or a specified group of such entities."

This constant $n_{1}$ has no agreed international name. It would be the responsibility of the relevant International Committees to agree a name. Possibly something like "elementary constant of amount of substance" or "elementary counting constant" would be appropriate. However, this note will refer to it simply as " $n_{1}$ ".

The relationship between the mole and $n_{1}$ can be written in the following way:

$1 \mathrm{~mol}=\Lambda_{\mathrm{A}} n_{1}$,

where $\Lambda_{\mathrm{A}}$ is the dimensionless quantity known as the Avogadro number. Following the May 2019 reforms [1] in the international system of measurement, the Avogadro number $\Lambda_{\mathrm{A}}$ is now specified exactly as:

$\Lambda_{\mathrm{A}}=6.02214076 \times 10^{23}$.

For the avoidance of confusion, note that the Avogadro constant $N_{\mathrm{A}}$ is a different physical quantity given by

$N_{\mathrm{A}}=\Lambda_{\mathrm{A}} / \mathrm{mol}=6.02214076 \times 10^{23} \mathrm{~mol}^{-1}$.

Comparison of Eqs. (1) and (3) shows that, in fact

$N_{\mathrm{A}}=1 / n_{1}$.

Hence, any physical formula that currently involves $N_{\mathrm{A}}$ could in principle be re-written in terms of $n_{1}$, although obviously it would be unconventional to do so, at present. What this does show, however, is that (now that $n_{1}$ has been formally defined) there is no need to introduce the Avogadro constant into the detailed discussions in the present note.

One next needs a convention about atomic-level units of amount of substance. The convention proposed here is that, in general discussions, the word "entity" should be used as the atomic-level unit of amount of substance, so we would have

$n_{1}=1$ entity.

In any specific context, where one is counting a specific entity such as an atom, it should be allowable to replace the word "entity" by the name of the entity being counted.

In practice, the use of the word "molecule" as if it were an atomic-level unit of amount of substance already occurs in chemical literature, particularly in discussion of various forms of molar energy, where "eV/molecule" is sometimes used as a more informative unit than " $\mathrm{J} / \mathrm{mol}$ ". In discussions of field evaporation theory, the words "atom" and "ion" are already sometimes used as if they were atomiclevel units of amount of substance. 
At some future time, it would possibly be useful to have a shorter defined symbol for the word "entity" when this is treated as an atomic-level unit of amount of substance, but use of the full word is sufficient for present purposes.

\subsection{Proposed Alternative Name for the Quantity Amount of Substance}

Techniques such as field ion microscopy are able to image the individual top-layer atoms in a crystal facet, and there is sometimes a need to quantify how many atoms are visible. For example, consider the case where there are three tungsten atoms visible in the topmost atomic layer. Macroscopic 7-dimensional language would be something like: "The amount of substance of tungsten atoms visible is 3 atoms", or possibly "The amount of tungsten atoms visible is 3 atoms". An alternative statement is that: "The count of tungsten atoms visible is 3 atoms". In the author's view, this alternative statement is easier to understand.

It is thus proposed that the term "count" should be used as an alternative name for the quantity "amount of substance", particularly in atomic-level contexts or in any other context where the phrases "amount of substance" or "amount" are linguistically clumsy. The meaning of the phrase "The count of" is self-evident, and there seems little possibility of misunderstanding. In the international system of measurement, there are already situations where alternatives are recognised-for example, the "Dalton" and the "unified atomic mass unit" are recognised alternative names for the atomiclevel unit of mass.

It is not being proposed that the name "amount of substance" (or the shortened version "amount") should be discontinued,--merely that "amount" and "count" should be seen as equivalent alternatives, with one or the other being clearer in a given linguistic context.

Of course, another alternative statement would be: "The number of tungsten atoms visible is 3".

But this is a statement made in 6-D terminology, and-for reasons that should become obvious below-I am trying to move away from the use of 6-D terminology.

An advantage of using the term "count" (rather than "amount") is that there is a linguistic imperative to specify precisely what entity is being counted. Thus, in the case of hydrogen molecules, the phrase "amount of hydrogen" is linguistically acceptable, but the phrase "count of hydrogen" is not. For linguistic acceptability, one needs to say "count of hydrogen molecules".

In what follows below, I implement the convention of using the term "count" as an alternative name for the quantity "amount of substance". In practice, the term "count" is already in informal scientific use; in particular, it is sometimes used to label the vertical axis of mass spectra.

\section{Application to Field-Evaporation Theory}

\subsection{Statement of 7-Dimensional Theory}

To implement a consistent 7-dimensional (7-D) discussion of the theory of field evaporation (FEV) from a material surface (or similar theories), several derived "7-D" quantities need to be defined.

Surface concentration $(\sigma)$ is: "the count of entities per unit area of the relevant surface" (dimension: $\mathbf{N L}^{-2}$ ). FEV flux $(R)$ is "the count of entities field evaporated per unit time" (dimension: $\mathbf{N T}^{-1}$ ).

FEV flux density $\left(J_{n}\right)$ is "the count of entities field evaporated per unit time per unit area of the relevant surface" (dimension: $\mathbf{N L}^{-2} \mathbf{T}^{-1}$ ).

Note that in the wider scientific literature there is ambiguity over the meaning of the term "flux", with some users, in some contexts, preferring to use this term to mean "flow per unit time per unit area", rather than "flow per unit time". The use here is a 7-D version of that in a recent International Standard [6], and aligns better with the well-accepted use of the electrical terms "current" and "current density".

Under normal circumstances, FEV is a thermally activated process, with an Arrhenius-type FEV rate-constant $k$ given by

$k=A \exp \left[-Q / k_{\mathrm{B}} T\right]$

where $Q$ is the FEV activation energy, $k_{\mathrm{B}} T$ is the Boltzmann factor, and $A$ is the FEV rate-constant pre-factor. This rateconstant $k$ has dimension $\mathbf{T}^{-1}$ and is measured in $\mathrm{s}^{-1}$.

When FEV takes place from a specimen/emitter, only the most protruding surface atoms (which are subject to particularly high surface fields) are at high risk of FEV. Hence, the FEV flux is given by

$R=n_{\mathrm{hr}} k_{\mathrm{hr}}=n_{\mathrm{hr}} A_{\mathrm{hr}} \exp \left[-Q_{\mathrm{hr}} / k_{\mathrm{B}} T\right]$,

where $n_{\mathrm{hr}}$ is the count of atoms at high risk of FEV, and the subscript "hr" is used to label the "high-risk" values of $k, A$ and $Q$. The factor $\left(n_{\mathrm{hr}} A_{\mathrm{hr}}\right)$ is sometimes called the FEV flux pre-factor.

Correspondingly, the FEV flux density $J_{n}$ is given by

$J_{n}=\sigma_{\mathrm{hr}} k_{\mathrm{hr}}=\sigma_{\mathrm{hr}} A_{\mathrm{hr}} \exp \left[-Q_{\mathrm{hr}} / k_{\mathrm{B}} T\right]$,

where $\sigma_{\mathrm{hr}}$ is the surface concentration of atoms at high risk of FEV (and could be called the "FEV surface concentration").

The parameters $n_{\mathrm{hr}}$ and $\sigma_{\mathrm{hr}}$ (and the related 6-D parameters) are all functions of field and temperature, and also depend on the details of emitter shape. They are sometimes roughly approximated as constants, for the purposes of making order-of-magnitude estimates of flux and flux density (e.g., see [3]); detailed evaluation of their values, and those 
of $k_{\mathrm{hr}}$, would involve very complicated processes of integration and averaging over the emitting surface (e.g., see [7]).

\subsection{Comparison with 6-Dimensional Theory}

The 6-dimensional (6-D) equivalent of the "count of entities" $(n)$ is the "number of entities" $(N)$ given formally by

$N=n / n_{1}$.

The other 6-D equivalents are also formally obtained by dividing by $n_{1}$, as shown in Table 1 .

The 6-D equivalent of Eq. (7) is:

$\Phi=N_{\mathrm{hr}} k_{\mathrm{hr}}=N_{\mathrm{hr}} A_{\mathrm{hr}} \exp \left[-Q_{\mathrm{hr}} / k_{\mathrm{B}} T\right]$,

where $N_{\mathrm{hr}}\left[=n_{\mathrm{hr}} / n_{1}\right]$ is the number of atoms at high risk of FEV. The quantity $\Phi$ is commonly called the field evaporation rate, but the other 6-D quantities in Table 1 do not have well-established names.

There is also a 6-D equivalent of Eq. (8), but this is not widely used.

\subsection{Discussion in the Context of Field Evaporation Theory}

A problem with the name "field evaporation rate" is that the term "rate" is used more generally as a name for several different properties and quantities. In particular, confusion can occur (and often has occurred) between FEV rate $\mathrm{d} N / \mathrm{d} t$ and FEV-rate-constant $k_{\mathrm{hr}}$, both of which are measured in $\mathrm{s}^{-1}$. This has not infrequently resulted (even in specialist textbooks) in the statement of a defective equation for FEV rate in which the quantity $N_{\mathrm{hr}}$ (number of atoms at high risk of field evaporation) is omitted. A mistake of this kind is more difficult to make in a 7-D system, because FEV flux and FEV rate-constant have distinctively different names and different ISQ dimensions, and hence different units. The probable avoidance of a mistake of this kind (writing down a defective FEV equation) is a useful feature of a 7-D approach.

A significant practical obstruction to the adoption of the 7-D system proposed here is that it involves a change in deep-seated linguistic habits, from saying "number of atoms (etc.)" to saying "count of atoms (etc.)." It is also necessary to remember that (if, say, three atoms are visible in a specified location), then correct usages are:

6-D: The number of atoms visible is 3 .

7-D: The count of atoms visible is 3 atoms.

In the 7-D statement, the word "atom" is being used as an atomic-level unit of amount of substance (i.e., "count"), and is thus an "atomic-level counting unit".

My view is that this is an "initialisation problem", and that with attention and practice, this obstruction could be overcome relatively rapidly.

\section{Some Background Linguistic Issues}

This discussion also needs to be seen against the background of some linguistic issues relating to how the process of counting is dealt with in English linguistics.

The view of the author is that there are some words in English (of which "dozen" and "gross" are the most prominent examples) that are only used in the context of counting, and are not numbers in the sense that "12" and "144" are. In principle, words such "dozen" and "gross" could be given respectable scientific definitions by means of equations such as the following:

1 dozen $\equiv 12 n_{1}=12$ entities,

1 gross $\equiv 144 n_{1}=144$ entities.

When such words are used, there is a presumption that the nature of the entity being counted will be specified, either directly or implicitly. I have long considered [8] "dozen" and "gross" to be the names of linguistic entities that I call counting units. Brief consultation of Wikipedia suggests that use of the term "counting unit" in this sense has been independently adopted by many writers.

It is quite clear that a counting unit of this kind is not a "number" in the ordinary mathematical sense of the word. This can easily be established by comparing the sentences:

"In the queue, you are in position 12."

"In the queue, you are in position dozen."
Table 1 To illustrate the relationships between the 7-D quantities and the corresponding 6-D quantities

\begin{tabular}{lll}
\hline Property & 7-D quantity & 6-D quantity \\
\hline How many atoms (etc.) of interest & $n$ & $N=n / n_{1}$ \\
How many atoms (etc.) per unit area of emitting surface & $\sigma$ & $\mathrm{d} N / \mathrm{d} A=\sigma / n_{1}$ \\
How many atoms (etc.) field evaporated (etc.) per unit time & $R$ & $\Phi=R / n_{1}$ \\
$\begin{array}{l}\text { How many atoms (etc.) field evaporated (etc.) per unit time per unit } \\
\text { area }\end{array}$ & $J_{n}$ & $\mathrm{~d} \Phi / \mathrm{d} A=J_{n} / n_{1}$ \\
\hline
\end{tabular}


The first of these sentences is correct English, but the second is not. This shows that the word "dozen" is not the name of an ordinary mathematical number, but is a different kind of linguistic object.

Although the position is not entirely clear-cut in all cases, and some uses are specialist (e.g., "century") there are in English many words that are best regarded as counting units, or alternatively can be used as counting units. As well as "dozen" and "gross", these include: pair, triple, six-pack, score and century.

The view of the author is that the mole, also, can be described as a (very large) counting unit. Following the May 2019 reforms, as indicated above, the unit "the mole" can now be defined exactly by an equation equivalent in form to (11) and (12), namely

1 mole $\equiv 6.02214076 \times 10^{23} n_{1}=6.02214076 \times 10^{23}$ entities .

More generally, the view of the author is that the linguistic/conceptual category of "counting unit", and the distinction between "counting unit" and "number", are underrecognised--both in English-language linguistics and in formal documents associated with the international system of measurement.

\section{Possible Implications for the International System of Measurement}

\subsection{Atomic-Level Constants and Units}

Briefly, what the May 2019 reforms have done is to: (a) remove the remaining physical artefact (the international prototype of the kilogram) from the international system of measurement; (b) define exactly the numerical relationships between several SI units and the corresponding atomic-level quantities; and (c) make definitions of all other SI units to be in terms of fundamental physical processes and fundamental physical constants. A detailed description of the reforms can be found in [1].

Since the system has now been put on a basis that is built on atomic-level processes, it now seems timely to begin to look again at the issue of how to consistently and easily discuss atomic-level phenomena within the framework of the international system. In order to do this, it would be useful to have formally defined atomic-level constants and defined atomic-level units (and associated defined symbols) for each of the three "extensive" quantities: mass, electric charge and amount of substance. The present situation is summarised in Table 2.

At present (July 2020), the items in boxes 1-6 are defined by international agreement, and the items in boxes 10 and 11 are (what appear to be) reasonable and obvious suggestions that have been made in the present note. For the remaining boxes, there are no internationally agreed solutions. Hopefully, all these remaining boxes will eventually be completed as a result of international agreements, but it is outside the scope of this note to make suggestions.

It would also be helpful to eventually have internationally agreed names and symbols for all of the ratios $\left(1 \mathrm{~kg} / m_{\mathbf{u}}\right)$, $(1 \mathrm{C} / e)$ and $\left(1 \mathrm{~mol} / n_{1}\right)$. As things stand at present, only the ratio $\left(1 \mathrm{~mol} / n_{1}\right)$ has an agreed name (the Avogadro number), but there is no agreed symbol for it.

It would also be helpful (particularly in contexts such as mass spectrometry) to eventually have an agreed comprehensive nomenclature system for dealing with ratios of atomiclevel constants, such as $\left(e / n_{1}\right)$ or $\left(e / m_{\mathrm{u}}\right)$, but it is outside the scope of this note to discuss these issues further.

\subsection{The Language of Counting}

In the new (2019) "SI brochure" [1], the words "count", "counting" and "counting unit" do not appear in the discussion of the physical quantity "amount of substance". Rather, the following statement is made.

\section{The mole}

The mole, symbol mol, is the SI unit of amount of substance. One mole contains exactly $6.02214076 \times 10^{23}$ elementary entities. This number is the fixed numerical value of the Avogadro constant, $N_{\mathrm{A}}$, when expressed in the unit $\mathrm{mol}^{-1}$, and is called the Avogadro number. The amount of substance, symbol $n$, of a system is a measure of the number of specified elementary enti-

Table 2 Atomic-level constants and units

\begin{tabular}{llll}
\hline & Mass & Electric charge & Amount of substance (“count”) \\
\hline Atomic-level constant: name & $\begin{array}{l}\text { 1. Unified atomic } \\
\text { mass constant }\end{array}$ & 5. Elementary (positive) charge & 9. No agreed name \\
Atomic-level constant: symbol & 2. $m_{\mathrm{u}}$ & 6. $e$ & 10. $n_{1}$ \\
Atomic-level unit: name & 3. Unified atomic & 7. No specific name & 11. Entity \\
& mass unit, OR & & 12. No agreed symbol \\
\hline
\end{tabular}


ties. An elementary entity may be an atom, a molecule, an ion, an electron, any other particle or specified group of particles.

More generally, the 2019 SI brochure appears to envisage a continuation of the present system whereby the counting of large numbers of specified entities is done by means of the physical quantity "amount of substance" and the unit the mole, but the counting of small numbers of specified entities is done by means of the dimensionless quantity "number of specified entities".

For reasons of historical continuity, a definition of the general form given above, and the continuation of this twomethod general system may be necessary at this stage. However, at some future stage, when ideas relating to the process of counting are more widely appreciated, it may be that a general approach similar to that proposed above for field evaporation theory would have advantages, particularly in a world where nanotechnology and atomic-level manipulations are becoming increasingly important. The key feature of such an approach would again be that the counting of both large and small numbers of specified entities would be done in terms of a single physical quantity (the present "amount of substance"), but that the counting of small numbers would be facilitated by the definition of an atomic-level unit of amount of substance (the present "entity", as discussed above).

In such a future scenario, a more direct definition of necessary concepts could be formulated, perhaps something along the following lines.

The amount of substance (or count) of a system measures how many specified entities the system contains, in terms of a count of these entities. The specified entity may be an elementary entity, such as an atom, a molecule, an ion or an electron, or a specified group of elementary entities.

The mole, symbol mol, is the SI unit of amount of substance (or count). The mole is a large counting unit exactly equal to $6.02214076 \times 10^{23}$ specified entities. This number is called the Avogadro number.

For counting small numbers of specified entities, the term "entity" (or the name of the specified entity) is used as an atomic-level unit of amount of substance (or count).

As with field evaporation theory, a general merit of this approach would be that a single set of (7-D) definitions, relationships and equations can be used to describe both of: (a) counting situations involving large numbers of specified entities; and (b) counting situations involving small numbers of specified entities. This would replace the present need to use one set of (7-D) equations for large numbers, but a different set of (6-D) equations for small numbers.
An approach of this kind might be generally useful in the context of atomic-level rate theory.

A particular merit of the above form of definition is that it avoids use of the parameter "Avogadro constant". The Avogadro constant $N_{\mathrm{A}}$ was introduced as part of the $1970 \mathrm{~s}$ reforms that resulted in the modern ISQ, but (in the author's experience) has been responsible for many misunderstandings, particularly at high-school level and amongst nonexpert users of the concept of amount of substance.

This author is also of the view that introducing the terms "count" and "counting unit", into formal definitions relating to the concept of amount of substance, would be likely to make it easier to teach this concept at high-school level-particularly when associated ideas, such as "counting by weighting" are also used.

\section{Summary}

It is argued in this note that basic theories of field evaporation and field desorption are best formulated using a socalled 7-dimensional theoretical approach, based on using the quantity "count of atoms" (and related quantities) rather than "number of atoms" (and related quantities). Key to this are the introduction of a basic constant $n_{1}$ equal to " 1 entity" and the introduction of the term "count" as an alternative name for "amount of substance". This 7-D approach means that the same equations can be used for small as for very large "counts" of atoms (or other specified entities). Also, the use of 7-D, rather than 6-D, equations and terminology makes it less likely that basic mistakes will be made in formulating field evaporation theory.

It is also argued that, now the May 2019 reforms to the international system of measurement are in place, and with the growing importance of nanotechnology, attention needs to be given to the development of a comprehensive system of nomenclature relating to atomic-level constants and units for the three ISQ "extensive" quantities, namely mass, electric charge, and amount of substance.

It is also argued that, in the longer term, there might be merit in reformulating general scientific approaches to the process of counting and to the treatment of the physical quantity "amount of substance", in order to make greater use of the concepts of "counting" and "counting unit".

Open Access This article is licensed under a Creative Commons Attribution 4.0 International License, which permits use, sharing, adaptation, distribution and reproduction in any medium or format, as long as you give appropriate credit to the original author(s) and the source, provide a link to the Creative Commons licence, and indicate if changes were made. The images or other third party material in this article are included in the article's Creative Commons licence, unless indicated otherwise in a credit line to the material. If material is not included in the article's Creative Commons licence and your intended use is not 
permitted by statutory regulation or exceeds the permitted use, you will need to obtain permission directly from the copyright holder. To view a copy of this licence, visit http://creativecommons.org/licenses/by/4.0/.

\section{References}

1. Bureau International des Poids et Mesures (2019) The international system of units (SI), 9th edn. BIPM, Sèvres

2. Gault B, Moody MP, Cairney JM, Ringer SP (2012) Atom probe microscopy. Springer, New York

3. Miller MK, Forbes RG (2014) Atom probe tomography: the local electrode atom probe. Springer, New York

4. Moors M, de Bocarné TV, Kruse N (2007) Catal Today 124:61-70

5. Che F, Gray JT, Ha S, Kruse N, Scott SL, McEwen J-S (2018) ACS Catal 8:5153-5174
6. International Standards Organization (2013), International standard ISO 18115-1:2013(E): surface chemical analysis-vocabulary-Part 1: general terms and terms used in spectroscopy. ISO, Geneva. Electronic version available (20 July 2020) via cover page at: https://www.npl.co.uk/surface-technology/standardis ation-traceability

7. Gruber M, Vurpillot F, Bostel A, Deconihut B (2011) Surface Sci 605:2025-2031

8. Forbes RG (1977) Educ Chem 14:124

Publisher's Note Springer Nature remains neutral with regard to jurisdictional claims in published maps and institutional affiliations. 